\title{
An Educational Revolution and Innovative Technologies: The Role of Simulation
}

\author{
Gul Pinar \\ Nursing Department, Faculty of Health Science, Yıldırım Beyazıt University, Ankara, Turkey (Formerly) \\ Email: gpinar_1@hotmail.com
}

How to cite this paper: Pinar, G. (2020). An Educational Revolution and Innovative Technologies: The Role of Simulation. Creative Education, 11, 2218-2232.

https://doi.org/10.4236/ce.2020.1111162

Received: September 19, 2020

Accepted: November 10, 2020

Published: November 13, 2020

Copyright (c) 2020 by author(s) and Scientific Research Publishing Inc. This work is licensed under the Creative Commons Attribution International License (CC BY 4.0).

http://creativecommons.org/licenses/by/4.0/

\begin{abstract}
Simulation based education (SBE) reemerged an important part of lifelong learning, which is defined as the imitation of an actual case, event or behavior. There has been increased interest in using medical simulation due to lacking of practice, changing learning environment, increasing attention to human error, patient safety, communication problem, personal rights and freedoms. This approach has created a "tipping point" in the transition to simulation education as an effective tool based on competency, which is regarded as revolutionary change. SBE provides unique opportunities for learners to fill the gap between theory and practice as a teaching strategy in a safe environment. In fact, SBE is not new; it has long been a part of the education system across all fields such as military, aviation, nuclear power generation, business, medicine, and computer science to maintain safety, to gain experience and to enhance evaluation of performance. However, high-fidelity simulation is a relatively new concept providing multi-task scenarios and many feedback options due to the increasing degree of realism in medical education. In this paper, the importance of simulation beyond just technology, wide variety of categories of simulations that range from simple to sophisticated regarding providing knowledge and skill after practices in the learning environment is discussed, and the multidimensional benefits and limitations of simulation are also mentioned. This article presents a general overview of SBE that will hopefully help to generate interest in using simulation.
\end{abstract}

\section{Keywords}

Simulation, Trend Technologies, Innovative Practicing, Education

\section{Introduction}

Literature highlights the increasing challenges educators face in preparing learners for the real world. Recent changes in the culture of education with rapid 
advances in technology have focused on inadequacy in the learning experiences, and emphasized the need more intensive experiential model of learning and a safe environment designed to closely real situations. Various educational strategies are tested in order to enhance skills and knowledge in education by organizations with increasing the demand for quality around the world. Heath-care education program must be innovative and devise strategies that optimalize the learning in knowledge, skills, and attitudes, including professionalism, leadership, and communication in conjunction with safe patient outcomes. Simulation is becoming popular as a valuable tool in providing innovative learning experiences that bridge the gap between theory and practice in many fields such as business, computer industries, aviation, military, including medicine training (Meakim et al., 2013; Palaganas et al., 2015; Drews \& Bakdash, 2013; Berregan, 2014). Many professional tasks and high quality evaluation can be performed at the same time with simulation. It is necessary to know which tasks the simulators imitate, at which level of the skill is effective, which skills contribute more to learning. Simulation-based education (SBE) begun to be gradually introduced into medical areas like public health, critical care, obstetrics, emergency, internal and surgery medicine (Nestel et al., 2013; Aebersold et al., 2012; Paige \& Morin 2013; Levine et al., 2013). SBE is now recognized as an effective strategy that prepares healthcare professionals for the complex issues in the healthcare environment, changing the direction of education and learning style. Despite the demonstrated value of the approach to using simulation for ongoing patient-centered care still has not been completely integrated into all health care settings (Shah et al., 2013; Bilotta et al., 2013; Jefferies, 2007).

The aim of this paper is to describe the process of the simulation pedagogy, the role of simulation, the variety of simulation methods, and framework helping understand utilization of SBE in health care practices.

\section{What Is SBE?}

Simulation is an innovative teaching strategy to mirror real situation with guided immersive experiences in a fully interactive. This experience has been identified as essential components of evidence based learning, training, researching, and evaluating process in both academic and clinical setting. When administered correctly, SBE is shown as a realistic, safe, flexible, and cost-effective method if utilized properly that can be used to strengthen routine practices before exposed the real patients (Palaganas et al., 2015; Nestel et al., 2013). In fact, this trend is not limited to healthcare education system alone. Hospital management systems are also beginning to encounter utilization SBE practice by implementing evidence-based protocols and guidelines to improve the quality of care in their clinical setting. For the successful use of the SBE, its procedures need to be fully integrated into healthcare routine practice. This opportunity provides better outcomes in staff performance by analyzing unpredictable mistakes like an actual critical case (Aebersold et al., 2012). 


\section{What Is the Change in Health Care?}

Health education and practice system is radically changing in many fields around the world. The pedagogy of simulation has still constantly evolved, rapidly gaining acceptance and adopts the pace of developing medical technologies, increasing cultural diversity and globalizing education in health care delivery (Paige \& Morin, 2013). The new generation is problem-based, and prone to active learning in a variety of specialized roles, and legislation as an expert, care provider, consultant, leader, educator, and researcher. Education will naturally change to meet these new demands for practice. For this reason, health professionals not only need to master knowledge and procedural skills but also the ability to effectively communicate with patients, relatives, and other health care providers, as well as coordinate various cases. In fact, the change came about through the healthcare reform and involvement of the research results regarding learner performance (Shah et al., 2013). Current studies highlight the increasing challenges educators face a problem of how to provide meaningful clinical learning experiences and respond to the growing need for health care services that help students increase self-evaluation, particularly regarding the development of clinical judgment and critical thinking (INASCL, 2011). Thus, an innovative approach has been sought in order to meet the need for providing health services in a more humanistic approach. Looking from another perspective, patients may not desire to be "guinea pigs" of clinical education, and they want competent healthcare professionals to protect themselves from trials and errors, because they have no tolerance in this issue. There is also, on the other hand, an obligation to provide optimal treatment and to ensure patients' safety. Safety concept is important too for hospital management as one of their key performance indicators. Healthcare organizations began to take on more important roles within health system and they were considerable interest in preventing malpractice cases and provision of heath care (Shearer, 2013; Khala, 2014).

In the past, professionals believed that it was enough for learners to provide a variety of in-class clinical experiences to achieve certain competence. Nowadays, the professional SBE with a new technique, equipment and real environment offer unique opportunities for complex, and critical situations (Clarke et al., 2014; Valadares \& Magro, 2014). In addition, it has been realized that simulation as a dynamic method has the potential to revolutionize for competency-based learning styles of the new generation. Thus, simulation began to be widely accepted in the healthcare system due to overcome faculty and preceptor shortages, and lack of clinical sites. In the earlier times of medicine education, simple form of simulation was already being applied in the form of case scenarios and the use of case presentations. In fact, simulation has begun to change significantly with mantra "See one. Do one. Teach one". Today educators are concerned how trainees acquire the clinical experience in simple to highly complex technical skills. In this sense, health-care educators and providers need more information about how to best optimize simulation as a strategy in competency of 
knowledge, skill and behaviors (Hamstra et al., 2014; Mikasa et al., 2013).

\section{Why Is SBE So Crucial?}

SBE program would be a long-term investment in medical education, connecting theory with practice and allowing learners to acquire variety of skills through deliberate practice without causing harm (Kim et al., 2016; Cheng et al., 2015; Hayden et al., 2012). The most importantly of SBE is ensuring efficient allocation of resources, minimizing of number readmission in the hospital, preventing prolongation of hospitalization, providing cost reduction, and elimination of preventable adverse medical events in health care system (Couto et al., 2015; Cumin et al., 2013). The power of simulation is that it gives "fidelity" and "permission to fail" in a safe interactive environment, so students learn their mistakes, which leads to better patient outcomes. Simulation has also become more important as it shortens the working hours of the trainees by facilitating reliability learning. In particular, experiential learning of uncommon or rare situation is of great value, whereas this was quite difficult process to learn in healthcare settings (Foronda et al., 2013; Motola et al., 2013; McMullen et al. 2016). Notably, literature reflects that the use of SBE has proven to be an effective teaching and learning method to provide the opportunity for repetitive practice chance and immediate feedback. SBE with student-centered also offers a unique opportunity to educate people for master the skills of judgment, competency, self-confident, communication, delegation, conflict management, decision-making, ethical dilemmas and critical thinking skill without jeopardizing people safety using simulators. Also, it can be used to investigate errors in health-care before a sentinel event occurs (Richardson \& Claman, 2014; Nimbalkar et al., 2015; Ringel et al., 2015; Pinar \& Dogan, 2013; Schmidt et al., 2013; So et al., 2019; Tawalbeh \& Tubaishat, 2014). All of these positive outcomes eventually led the Accreditation Council for Graduate Medical Education (ACGME) to support and motivate the use of simulation in medical arena as an education and rigorously assessment process, which is become arts of the redesign of medical curriculum (ACGME, 2020). The success of SBE has led to the acceptance of simulation techniques and embraces the advancing tools and creative resources in other fields. Hence, it offers a great part of the solution to fill the educational gaps in various fields. The future of SBE in achieving expected goals depends on the technical, financial and political interventions of centralized system accrediting agencies, and the competency and effort of the health care simulation associations around the world (Palaganas et al., 2015; Nestel et al., 2013).

\section{What Are the Benefits of SBE?}

SBE is proved to have lots of long and short-term benefits increasing standardization in a dynamic learning environment, all of which can help guarantees experience on improving professional competency without putting patients at risk. SBE helps learners prepare to deal with unanticipated medical events and the 
ability to prioritize tasks under pressure in a controlled environment that are difficult to obtain in real life (Hart, 2017; Proctor \& Campbell-Wynn, 2014).

This experience can be expanded to community health practices like disaster planning. SBE reduces the risk of clinical errors using immediate feedback associated with learning outcomes on the specific situation with the opportunity to repeatedly apply clinical scenarios. Errors can be detected by effective protocols following all simulation exercises while away from actual clinical practice (Watson et al., 2012; Rudolph et al., 2014). Some of the advantages of SBE are listed in Table 1.

When health care providers of different disciplines train separately, it may be difficult to integrate their capabilities. Effective multidisciplinary teams must always have good communications and leadership sharing behavior, which can help ensure patient safety. Learners are made aware of their synergistic roles working with other team members, thus it has the potential to create lasting and sustainable behavior and culture change. The complexity of scenarios is easy to integrate into the simulation process taking into account the learner needs and selected sessions can be tailored or calibrated to target more than one learning strategy for clinical diversity or encouraging proactive learning (Michael et al., 2014; Zapko et al., 2018). SBE science is well researched and an approach based on educational outcomes and its design has focused on validated processes and evaluations. At the end of SBE sessions, learners and educators truly enjoy their SBE experience and typically request additional training opportunities and various resources to sustainable of program. According to the literature, simulation may reduce stress and anxiety of learners, which could provide effective learning and they were reluctant to participate because of the fear of making mistakes (Ferri et al., 2018; Quail et al., 2019; Cantrell et al., 2017). Despite of all of these advantages of simulation, training through simulation needs to be viewed as an

Table 1. Advantages of SBE.

\begin{tabular}{ll}
\hline Knowledge acquision/retention & Change attitude, value and behavior positively \\
Preventing of anxiety & Facilitating learning, shorten the learning period \\
Solving ethical dilemma & Improving workplace culture, cost-effective \\
Increasing patient satisfaction & Increasing learner/staff satisfaction \\
Reducing malpractice & Increasing patient outcomes \\
Safe, risk-free learning environment & Immersive, experiential and repeatedly practice \\
Guarantee the same quality of care & Competence and feedback for learner/educators \\
Inter-professional communication & Equitable technical and functional expertise \\
Displaying team-work & Encourage research leading to improvement \\
Promote leadership, executive & Problem-solving, delegation, consultation \\
Rapid decision making capacity & Provide self-confidence, self-efficacy \\
Critical-thinking, life-saving skills & Competency in simple, complex or rarely cases \\
Stress and crisis management & Optimal clinical judgment and criticized \\
\hline
\end{tabular}


alterative for learning in the clinical setting. SBE is not intended to replace the learning of real patients (Watson et al., 2012).

\section{What Are the Types of SBE?}

Simulation may raise the bar for facilitating patient safety. There is a wide range of simulator fidelities, which can be utilized to role-playing the selected scenarios from simple intervention to interdisciplinary complex problem management such as virtual-reality; screen-based simulator providing feedback about the learner's practice (Palaganas et al., 2015; Paige \& Morin, 2013). Simulators may allow advanced networking to be implemented remotely, low, moderate and high fidelity simulators, life-like human mannequin that breathes, blinks, talks, and has heart beat, pulse and lung sounds. Task trainers represent anatomical human body parts that are used to improving basic procedural skills and have limited responses capabilities. Low fidelity simulators (LFS) are partial or whole body mannequins that are used to train students in specific skills such as injections and catheterizations. Intermediate or medium-fidelity simulators (MFS) are computerized partial body mannequins that are used to teach skills such as assessment of lung sounds (Levine et al., 2013; Shah et al., 2013). High-fidelity simulation (HFS) is a highly technical, life-like human mannequin connected the computer system that control the physiological and physical reactions of the simulator such as audible chest movement, simulate various breath, heart, bowel and lung sounds; provide electrocardiogram waveforms, cardiac output, and pulse-oximeter readings. Additionally, it has palpable pulses, blinking eyes and even speech. The HFS modules with evidence-based clinical scenarios for training purposes are being able ensure real experiences and immediate feedback about knowledge, skills, and behaviors to student in a safe immersive environment (Shearer et al., 2013; Palaganas et al., 2015).

The other strategy is the use of standardized patients. A simulated patient is a person who has been trained to act like a patient with a particular disease. Standardized patients are thought how to answer questions; also they are provided a script so that they cannot deviate. The primary goals of standardized patient encounters are o standardize evaluation criteria, to offer consistent clinical experiences such as obtaining a health history, performing physical examination and consultations, conducting interviews, and developing a care plan (Nestel et al., 2013; Jefferies, 2007).

Simulator types are listed in Table 2. A mobile simulation has become more popular with many examples and can be high-impact mechanism and significant value to simulation training. Virtual reality includes video-games technology and integrates interactive computer simulation with psychomotor and cognitive learning experience in specific procedures such as amniocentesis, bronchoscopy, colonoscopy, intravenous catheter insertion, and intubation. Virtual reality also provides cues via sensory stimulation (hearing, touch, and visual) to evoke feelings of fidelity. Haptic (touch) systems create an illusion of having direct contact 
Table 2. Taxonomy and types of simulator.

\begin{tabular}{|c|c|}
\hline Types-taxonomy of simulator & Examples \\
\hline Cadavers/ Animal models & $\begin{array}{l}\text { Procedural training; Intravenous access, } \\
\text { suturing }\end{array}$ \\
\hline Peer-to-peer-role playing & $\begin{array}{l}\text { Collaboration between peers used to } \\
\text { master skills }\end{array}$ \\
\hline $\begin{array}{l}\text { High-tech-Screen-based procedural } \\
\text { simulators }\end{array}$ & $\begin{array}{l}\text { Portable computer programs, or the Web can be } \\
\text { conducted in the privacy of the learner's home or } \\
\text { office using their own personal computer. }\end{array}$ \\
\hline $\begin{array}{l}\text { LFS-basic procedures } \\
\text { MFS-static mannequin } \\
\text { In-situ-actual bed-site }\end{array}$ & $\begin{array}{l}\text { Pelvic examination, peace-maker, IV insertion, } \\
\text { episiotomy, pulse, heart and breath sounds } \\
\text { without the ability talk, chest or eye movement }\end{array}$ \\
\hline $\begin{array}{l}\text { HFS-fully-interactive mannequin-based } \\
\text { computer or tablets with automatic } \\
\text { response for neonatal-toddler-adult }\end{array}$ & $\begin{array}{l}\text { Ability to speak, move, breath, have blink, } \\
\text { lung sound, heartbeat, bleeding, pupillary reaction, } \\
\text { peripheral pulses, blood pressure, produce urine, } \\
\text { childbirth, CPR, ECG, seizure, hypoxic, } \\
\text { bronchoscopy, anesthesia, surgery }\end{array}$ \\
\hline $\begin{array}{l}\text { Part task trainers; a part of the anatomy, } \\
\text { plastic-based }\end{array}$ & $\begin{array}{l}\text { Anatomical models-wound dressing, intubation, } \\
\text { urinary catheterization, epidural placement, } \\
\text { occultation, USG }\end{array}$ \\
\hline $\begin{array}{l}\text { Virtual Reality and Haptic Systems-graphic } \\
\text { monitor and advanced human-computer }\end{array}$ & $\begin{array}{l}\text { Through } 2 \text { or } 3 \text {-D visual and audio, with the use } \\
\text { of an avatar (immersive experience). } \\
\text { Tomography scan images, laparoscopy-endoscopy }\end{array}$ \\
\hline $\begin{array}{l}\text { Standardized-simulated actors, } \\
\text { environment with real medical equipment }\end{array}$ & $\begin{array}{l}\text { Used in both immersive simulation-based training } \\
\text { sessions or to target specific knowledge, skills, or } \\
\text { attitudes, telling bad news, mock depression attack }\end{array}$ \\
\hline Integrated models-Hybrid simulations & $\begin{array}{l}\text { Combination of standardized patients and } \\
\text { part-task trainers, intensive care, } \\
\text { saturation measurement }\end{array}$ \\
\hline $\begin{array}{l}\text { Computer-based simulators } \\
\text { Game-based-tablet, smartphone }\end{array}$ & $\begin{array}{l}\text { Drug administration; uses mouse and keyboards } \\
\text { navigation for multiple practice, robotic surgery }\end{array}$ \\
\hline $\begin{array}{l}\text { Mobile simulation-off-site for } \\
\text { community outreach }\end{array}$ & $\begin{array}{l}\text { Disaster, intensive care, trauma, } \\
\text { emergency care skills }\end{array}$ \\
\hline
\end{tabular}

with the simulator, which are used extensively with surgical training such as laparoscopic and endoscopic surgery skill (Proctor \& Campbell-Wynn, 2014; Quail et al., 2016). In order to create an effective education electronic medical record with patient's histories, notes, lab and radiology results are utilized to manage the scenario. Computer-based simulator is promising without the restriction of place and time since it allows an objective computer-generated evaluation and enables self-learning (for example; pre-intra and post surgery training). Navigation options allow learner to tailor the learning to their needs with written documentation of the specific skill, including multiple-choice case scenario and performance tests. Hybrid simulations use a standard patient for the interactive learning of the scenario. For recording physical findings, being in labor or having a murmur can be used a manufactured partial body simulator to exhibit the required specific symptom (Shearer et al., 2013; Drews \& Bakdash, 2013). 


\section{How Can We Integrate SBE in to the Curriculum? Pearls and Pitfalls}

The simulation can be effectively integrated into the larger healthcare organizations with various phases in a formal manner, using a specific methodology. Many organizations have regularly developed their existing process or shaped new areas of focus using standard in providing their own progress and updating their mastery of certain practices (Shah et al., 2013). Despite the increasing use of SBE, some challenges initially have been noted in the integration of simulation into the curriculum. For long-term maintenance of the integration of SBE with constructive spirit, it is need to pay attention, cooperation, dedication, and patience to establish the facilities and support resources in all stages for effective interventions (Ringel et al., 2015; Schmidt et al., 2013). These difficulties are below as followed:

- Lack of ethnically diversity

- Lack of finance and partner

- Lack of information or academic preparation (undergraduate/postgraduate)

- Lack of role clarity, professionalism

- Lack of faculty or peer support or belief and defensiveness

- Lack of time to prepare for SBE activities

- Lack of clear communication and collaborative approach

- Lack of philosophical influences, values learning

- Lack of staff and heavy workload

- Lack of institute facilitates, space, clinical setting or equipment

- Lack of feedback

In fact, all of these challenges will be essential. In the process of integrating the simulation model into the curriculum, as a priority, standards of best practice simulation should be defined to provide further clarity and the same standardized experiences for all participants (Sho et al., 2019; Tawalbeh \& Tubaishat, 2014; Hart, 2017; Lee et al., 2016). Then, an integration team should be formed to manage and evaluate the process with proper framework. Choosing the framework in the simulation application is important in terms of providing a common perspective and language use for the application and creating consensus, but it also provides guidance for the integration process (Proctor \& Campbell-Wynn, 2014).

In Table 3, there are questions that can be evaluated during the integration process. The theoretical model consists of five major areas: teacher factors, student factors, educational practices, simulation design and outcomes. Design elements serve to evaluate the simulated activities such as objectives, support, problem solving, feedback, and fidelity. For true system integration, it includes analysis (based on needs and gap), planning of implementation with a guide protocol, development of resources, implementation of the plan, evaluation, revision of the plan and implementation (Jefferies, 2007). The integration team should assess staffing needs and be able to identify extra requirements regarding non-technical and technical skills. The function of the team is to create the plan 
Table 3. Questions about integration of simulation into the curriculum.

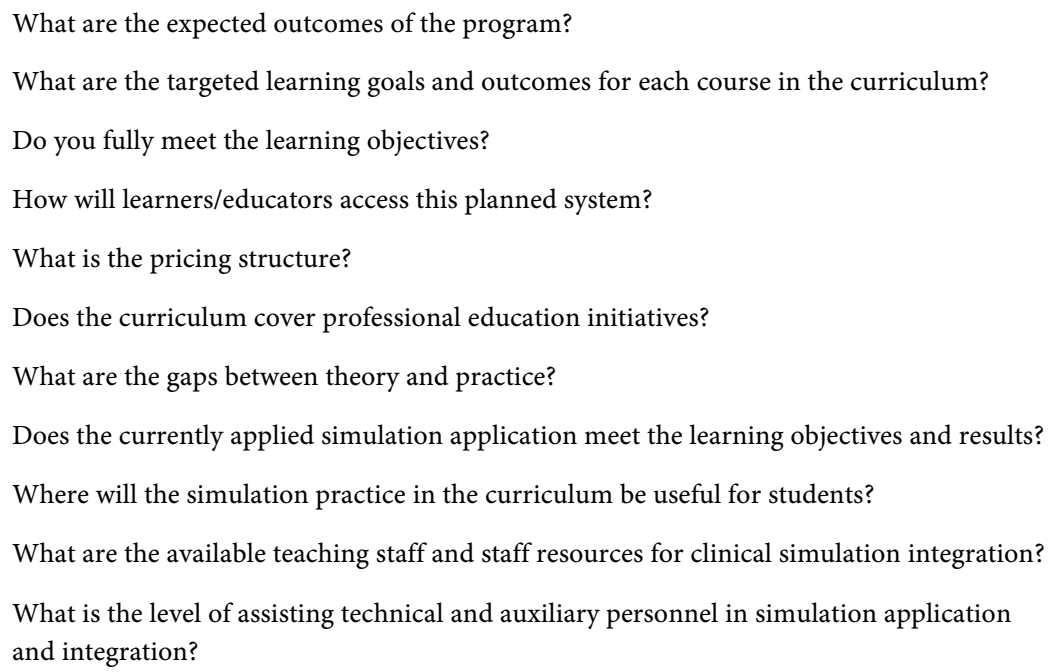

for the integration of simulation into the curriculum and provide the necessary guidance. These roles include determining a convenient location on the hospital or university campus for convenience of proximity, classifying technology according to the level of the simulation model, using computers and electronic devices, defining the barriers by determining the financial budget, managing the necessary equipment, and finally establishing the relevant policies (INASCL, 2011).

\section{Why Team Training in SBE?}

Educator can be designed entire simulation sessions for regulation purposes by using multidisciplinary feedback. Team members have to work together in a purposeful way (Couto et al., 2015). Furthermore, they usually come from separate disciplines with diverse educational preparation. Teamwork does not occur spontaneously, it has to be rehearsed and simulation is a unique tool with realistic design for training and evaluating process. For team members to fully engage in a simulation session, educators must support to learners by establishing a well-design environment (Motola et al., 2013). This approach is used to learn open relationship, to control of environment, to discuss management of critical situations, and to enhance patient outcomes. Choosing the framework in simulation application on culture of teamwork and their clinical competence assessment is important in terms of common perspective for creating consensus. Mutual support is also the essence of teamwork, it protects team members from work overload and poor task assignment that may reduce effectiveness and increase the risk of error (Drews \& Bakdash, 2013).

Developing teamwork strategies will contribute to structuring the professional skills such as leadership, communication, self-evaluation and corporation. In the previous studies, strong relationships have been shown to exist between teamwork and positive outcomes. Finally, there has been an increased focus on this 
topic with the purpose of enhancing patient safety (Berregan, 2014, Cumin et al., 2013; Schmidt et al., 2013). All of simulation organizations also have focused on teamwork clinical practices, which are slowly replacing more traditional didactic, lecture-based individual learning methods. As seen in Table 4, there are some techniques, which provides a standardized framework to teamwork and communicate for problem solving approaches (Couto et al., 2015; Hayden et al., 2012; Levine et al., 2013).

\section{How Can We Implementation of SBE?}

The implementation of simulation includes four stages; pre-briefing, briefing, scenario, and debriefing (Nestel et al., 2013; Aebersold et al., 2012). Well-planned the goals of the SBE program, style of train the trainees and moulage supplies, mode of simulation, proper scenario design based on theoretical background, and development suitable performance measurement (summative and formative) such as Likert style form, metric, checklist, and OSCE form to define the learners expectations, feedback, knowledge, skills and attitudes that show competency is defined and all supporting materials are produced (Berragan, 2014). All of these outcomes should be recorded to complete effective educational activities and to initiate discussion and to make sure that all learning objectives, including sociological fidelity were covered. Initially, a simulation group consisting of a maximum of 4 - 5 participants is created. Each participant in the group is given a role related simulation. Participants are received the pre-briefing

Table 4. Expected teamwork behaviors; SBAR, IPASS, and BATON.

\begin{tabular}{|c|c|}
\hline SBAR & Teamwork-communication behaviors \\
\hline Situation & What is happening with the patient? \\
\hline Background & What is the clinical current problem? \\
\hline Assessment & What do I think the problem is? \\
\hline Recommendation & What would I recommend? \\
\hline \multicolumn{2}{|l|}{ IPASS } \\
\hline Introduction & Introduce your role/job \\
\hline Patient & Identifiers; sex, age, location \\
\hline Assessment & Vital signs, symptoms, and diagnosis \\
\hline Situation & Current status, code, changes, response \\
\hline Safety & Critical lab values, allergies \\
\hline \multicolumn{2}{|l|}{ BATON } \\
\hline Background & Co-morbidities, medications, anamnesis \\
\hline Actions & What actions were taken required? \\
\hline Timing & Level of urgency and explicit timing \\
\hline Ownership & Who is responsible (nurse/doctor/...)? \\
\hline Next & What will happen next? Plan changes? \\
\hline
\end{tabular}


and read the entire simulation scenario includes purposing to perform various assessments, identify treatment needs, provide patient education and engage in effective communication (Paige \& Morin, 2013; Palaganas et al., 2015). Participants are explicitly asked to treat as if they were in a real situation during the simulation. The scenarios should be realistic, practical, and comprehensive and the educator should act as a facilitator providing cues when necessary. Scenarios would usually also have event triggers, environmental distractors, and supporting events. During simulation is broadcast on a projector for the rest of the class to watch. The practice of a scenario can be videotaped for immediate feedback to participants during the debriefing sessions (Aebersold et al., 2012; Rudolph et al., 2014).

After simulation scenario, the educator and the student should participate in an active debriefing. Video recording of the scenario is used to initiate discussion and to make sure that all learning objectives were covered (Meakim et al., 2013; Nimbalkar et al., 2015; Pinar \& Dogan, 2013). The learner's performance needs to be analyzed, summative and formative feedback and debriefing, respectively. Debriefing after a scenario is an important component of full-scale simulation. At the end of each debrief session, the facilitator highlights the learning goals and reinforced the importance of safety patient care. Poor management may lead to students questioning and decrease in satisfaction or realism. In addition, learner self-reflection has become a key component to contribute to the development of simulation training (Foronda et al., 2013, Watson et al., 2012; Cantrell et al., 2017).

\section{Conclusion and Recommendations}

SBE has opened up a realistic and multifaceted alternative strategy in medical education. For many advantages, the SBE as an authentic and interactive teaching method will remain an ethical imperative and spread quickly across the world. Many researches and evidence-based studies have shown that SBE is the potential of effective, a modern technique in improving learners' clinical knowledge, competence, attitudes, patient safety, and significantly reduces the learning time to reach the level of proficiency, and health care costs. Ultimately, SBE continues to evolve depending on overcoming issues related to technology, research, cost, faculty development, and cultural background, and redefine itself. Educators will have to adapt in order to create effective learning setting. The future is promisingly bright for SBE providing numerous possibilities to improve clinical education for educators and learners. It is inevitably thought that even greater steps will be taken in the future in achieving fundamental right regarding education and health issues.

Educators need to remember one important aspect; not all success in simulation may always relate to highly technical systems, but the right mix of various simulation experiences will also be effective in achieving positive results. Despite the increasingly wide spread use of simulation in health education, there are few evidence-based results that its use improves patient outcomes directly. 
Therefore, more randomized controlled clinical trials and more technically equipped methods are needed to identify components of fully validated to update performance indicators, and increase effectiveness on outcomes. In addition, it needs to focus on the multidimensional efforts to put into practice initiatives to eliminate unequal opportunities between regions and countries by producing more reasonably priced simulators. Health society organizations and higher education institutions should make arrangements for extensive capabilities and the development of clinical skills in educational environments and obtain sponsor support. Also, clinic and academic faculties must be done continuously the process of certification and accreditation to ensure standardization of professionals, and thus to reduce limited utility of SBE.

\section{Conflicts of Interest}

The author declares no conflicts of interest regarding the publication of this paper.

\section{References}

Accreditation Council for Graduate Medical Education ACGME (2020). Program Requirements for Graduate Medical Education in General; Policies and Procedures. https://acgme.org/Portals/0/PDFs/ab ACGMEPoliciesProcedures.pdf

Aebersold, M., Tschannen, D., \& Bathish, M. (2012). Innovative Simulation Strategies in Education. Nursing Research and Practice, 2012, Article ID: 765212. https://doi.org/10.1155/2012/765212

Berragan, L. (2014). Learning Nursing through Simulation: A Case Study Approach towards an Expansive Model of Learning. Nurse Education Today, 34, 1143-1148. https://doi.org/10.1016/j.nedt.2014.03.005

Bilotta, F. F., Werner, S. M., Bergese, S. D., \& Rosa, G. (2013). Impact and Implementation of Simulation-Based Training for Safety. Science World Journal, 7, Article ID: 652956. https://doi.org/10.1155/2013/652956

Cantrell, M. L., Meyer, S. L., \& Mosack, V. J. (2017). Effects of Simulation on Nursing Student Stress: An Integrative Review. Nursing Education, 56, 139-144. https://doi.org/10.3928/01484834-20170222-04

Cheng, A., Lockey, A., Bhanji, F., Lin, Y., Hunt, E. A., \& Lang, E. (2015). The Use of High-Fidelity Manikins for Advanced Life Support Training-A Systematic Review and Meta-Analysis. Resuscitation, 93, 1429. https://doi.org/10.1016/j.resuscitation.2015.04.004

Clarke, S., Horeczko, T., Cotton, D., \& Bair, A. (2014). Heart Rate, Anxiety and Performance of Residents during a Simulated Critical Clinical Encounter: A Pilot Study. BMC Medical Education, 14, Article No. 153. https://doi.org/10.1186/1472-6920-14-153

Couto, T. B., Kerrey, B. T., Taylor, R. G., FitzGerald, M., \& Geis, G. L. (2015). Teamwork Skills in Actual, in Situ, and In-Center Pediatric Emergencies: Performance Levels across Settings and Perceptions of Comparative Educational Impact. Simulation in Healthcare, 10, 76-84. https://doi.org/10.1097/SIH.0000000000000081

Cumin, D., Boyd, M. J., Webster, C. S., \& Weller, J. M. (2013). A Systematic Review of Simulation for Multidisciplinary Team Training in Operating Rooms. Simulation in Healthcare, 8, 171-179. https://doi.org/10.1097/SIH.0b013e31827e2f4c 
Drews, F. A., \& Bakdash, J. Z. (2013). Simulation Training in Health Care. Reviews of Human Factors and Ergonomics, 8, 191-234. https://doi.org/10.1177/1557234X13492977

Ferri, P., Rovesti, S., Magnani, D. et al. (2018). The Efficacy of Interprofessional Simulation in Improving Collaborative Attitude between Nursing Students and Residents in Medicine. A Study Protocol for a Randomized Controlled Trial. Acta BioMedica, 89, 32-40.

Foronda, C., Liu, S., \& Bauman, E. (2013). Evaluation of Simulation in Undergraduate Education: An Integrative Review. Clinical Simulation in Nursing, 9, 409-416. https://doi.org/10.1016/j.ecns.2012.11.003

Hamstra, S. J., Brydges, R., Hatala, R., Zendejas, B., \& Cook, D. A. (2014). Reconsidering Fidelity in Simulation Based Training. Academic Medicine, 89, 387-392.

https://doi.org/10.1097/ACM.0000000000000130

Hart, S. (2017). Today's Learners and Educators; Bringing the Generational Gaps. Teaching and Learning in Nursing, 12, 253-257. https://doi.org/10.1016/j.teln.2017.05.003

Hayden, J., Jeffries, P., \& Kardong-Edgren, S. (2012). The NCSBN National Simulation Study. Clinical Simulation in Nursing, 8, 407.

https://doi.org/10.1016/j.ecns.2012.07.070

Jefferies, P. R. (2007). Simulation in Nursing Education: From Conceptualization to Evaluation. New York: National League for Nursing.

Khala, R. (2014). Simulation in Nursing Education: An Evaluation of Students' Outcomes at Their First Clinical Practice Combined with Simulations. Nurse Education Today, 34, 252-258. https://doi.org/10.1016/j.nedt.2013.08.015

Kim, J. H., Park, J. H., \& Shin, S. J. (2016). Effectiveness of Simulation-Based Nursing Education Depending on Fidelity: A Meta-Analysis. BMC Medical Education, 16, Article No. 152. https://doi.org/10.1186/s12909-016-0672-7

Lee, J., Lee, Y., Lee, S., \& Baem, J. (2016). Effects of High-Fidelity Patient Simulation Led Clinical Reasoning Course: Focused on Nursing Core Competencies, Problem Solving, and Academic Self-Efficacy. Japan Journal of Nursing Science, 13, 20-28. https://doi.org/10.1111/jins.12080

Levine, A. I., DeMaria, Jr. S., Schwartz, A. D., \& Sim, A. J. (2013). The Comprehensive Textbook of Healthcare Simulation. Berlin: Springer Science \& Business Media. https://doi.org/10.1007/978-1-4614-5993-4

McMullen, M., Wilson, R., Fleming, M. et al. (2016). “Debriefing-On-Demand”: A Pilot Assessment of Using a "Pause Button" in Medical Simulation. Simulation in Healthcare, 11, 157-163. https://doi.org/10.1097/SIH.0000000000000140

Meakim, C., Boese, T., Decker, S. et al. (2013). Standards of Best Practice: Simulation Standard I: Terminology. Clinical Simulation in Nursing, 9, 3-11. https://doi.org/10.1016/j.ecns.2013.04.001

Michael, M., Abboudi, H., Ker, J., Shamim Khan, M., Dasgupta, P., \& Ahmed, K. (2014). Performance of Technology-Driven Simulators for Medical Students-A Systematic Review. Journal of Surgical Research, 192, 531-543. https://doi.org/10.1016/j.jss.2014.06.043

Mikasa, A. W., Cicero, T. F., \& Adamson, K. A. (2013). Outcome-Based Evaluation Tool to Evaluate Student Performance in High-Fidelity Simulation. Clinical Simulation in Nursing, 9, 361-367. https://doi.org/10.1016/j.ecns.2012.06.001

Motola, I., Devine, L. A., Chung, H. S. et al. (2013). Simulation in Healthcare Education: A Best Evidence Practical Guide. AMEE Guide No. 82. Medical Teacher, 35, 1511-1530. 
https://doi.org/10.3109/0142159X.2013.818632

Nestel, D., Watson, M. O., Bearman, M. L., Morrison, T. et al. (2013). Strategic Approaches to Simulation-Based Education: A Case Study from Australia. Journal of Health Specialities, 1, 4-10. https://doi.org/10.4103/1658-600X.110666

Nimbalkar, A., Patel, D., Kungwani, A., Phatak, A., Vasa, R., \& Nimbalkar, S. (2015). Randomized Control Trial of High Fidelity vs Low Fidelity Simulation for Training Undergraduate Students in Neonatal Resuscitation. BMC Research Notes, 8, Article No. 636. https://doi.org/10.1186/s13104-015-1623-9

Paige, J. B., \& Morin, K. H. (2013). Simulation Fidelity and Cueing: A Systematic Review of the Literature. Clinical Simulation in Nursing, 9, 481-489.

https://doi.org/10.1016/j.ecns.2013.01.001

Palaganas, J. C., Maxworthy, J. C., Epps, C. A., \& Mancini, M. E. (2015). Defining Excellence in Simulation Programs. Alphen aan den Rijn: Wolters Kluwer.

Pınar, G., \& Dogan, N. (2013). Improving Perinatal Patient Safety among Turkish Nursing Students Using Simulation Training. Social and Behavioral Sciences, 83, 88-93. https://doi.org/10.1016/j.sbspro.2013.06.017

Proctor, M. D., \& Campbell-Wynn, L. (2014). Effectiveness, Usability, and Acceptability of Haptic-Enabled Virtual Reality and Mannequin Modality Simulators for Surgical Cricothyroidotomy. Military Medicine, 179, 260-264. https://doi.org/10.7205/MILMED-D-13-00365

Quail, M., Brundage, S. B., Spitalnick, J., Allen, P. J., \& Beilby, J. (2016). Student Self-Reported Communication Skills, Knowledge and Confidence across Standardized Patient, Virtual and Traditional Clinical Learning Environments. BMC Medical Education, 16, Article No. 73. https://doi.org/10.1186/s12909-016-0577-5

Richardson, K. J., \& Claman, F. (2014). High-Fidelity Simulation in Nursing Education: A Change in Clinical Practice. Nursing Education Perspectives, 35, 125-127. https://doi.org/10.1097/00024776-201403000-00012

Ringel, N., Bürmann, B. M., Fellmer-Drueg, E., Roos, M., Herzog, W., \& Nikendei, C. (2015). Integrated Peer Teaching of Communication and Clinical Skills How to Train Student Tutors? Psychotherapie, Psychosomatik, Medizinische Psychologie, 65, 288-295. https://doi.org/10.1055/s-0034-1398549

Rudolph, J. W., Raemer, D. B., \& Simon, R. (2014). Establishing a Safe Container for Learning in Simulation: The Role of the Pre-Simulation Briefing. Simulation in Healthcare, 9, 339-349. https://doi.org/10.1097/SIH.0000000000000047

Schmidt, E., Goldhaber-Fiebert, S. N., Ho, L. A., \& McDonald, K. M. (2013). Simulation Exercises as a Patient Safety Strategy: A Systematic Review. Annals of Internal Medicine, 158, 426. https://doi.org/10.7326/0003-4819-158-5-201303051-00010

Shah, A., Carter, T., Kuwani, T., \& Sharpe, R. (2013). Simulation to Develop Tomorrow's Medical Registrar. The Clinical Teacher, 10, 42-46. https://doi.org/10.1111/j.1743-498X.2012.00598.x

Shearer, J. E. (2013). High-Fidelity Simulation and Safety: An Integrative Review. Journal of Nursing Education, 52, 39-45. https://doi.org/10.3928/01484834-20121121-01

So, H. Y., Chen, P. P., Wong, G. K. C., \& Chan, T. T. N. (2019). Simulation in Medical Education. Journal of the Royal College of Physicians of Edinburgh, 49, 52-57. https://doi.org/10.4997/JRCPE.2019.112

Tawalbeh, L., \& Tubaishat, A. (2014). Effect of Simulation on Knowledge of Advanced Cardiac Life Support, Knowledge Retention, and Confidence of Nursing Students in Jordan. Journal of Nursing Education, 53, 38-44. 
https://doi.org/10.3928/01484834-20131218-01

The INASCL Board of Directors (2011). Standards of Best Practice: Simulation. Clinical Simulation in Nursing, 7, 3-19. https://doi.org/10.1016/j.ecns.2011.05.005

Valadares, A. F., \& Magro, M. C. (2014). Opinions of Nursing Students on Realistic Simulation and the Curriculum Internship in Hospital Setting. Acta Paulista de Enfermagem, 27, 138-143. https://doi.org/10.1590/1982-0194201400025

Watson, K., Wright, A., Morris, N. et al. (2012). Can Simulation Replaces Part of Clinical Time? Two Parallel Randomized Controlled Trials. Medical Education, 46, 657-667. https://doi.org/10.1111/j.1365-2923.2012.04295.x

Zapko, K. A., Ferranto, M. L. G., Blasiman, R., \& Shelestak, D. (2018). Evaluating Best Educational Practices, Student Satisfaction, and Self-Confidence in Simulation: A Descriptive Study. Nurse Education Today, 60, 28-34.

https://doi.org/10.1016/j.nedt.2017.09.006 